\title{
EFEITO MÚTUO ENTRE O CRESCIMENTO DE NOVAS EMPRESAS E O DESENVOLVIMENTO DA SOCIEDADE BRASILEIRA: O PAPEL DA ATIVIDADE EMPRESARIAL NOS ESTADOS BRASILEIROS
}

\section{RESUMO}

Algumas tipologias jurídicas empresariais no Brasil podem favorecer ou inibir o desenvolvimento da sociedade, simultaneamente a trajetória desse desenvolvimento pode incentivar ou decair taxas de criação de novas empresas. Porém, essas relações são pouco investigadas na literatura brasileira. A pesquisa visou analisar os efeitos mútuos entre o crescimento de novas empresas (total e subdividido por tipos jurídicos) e o desenvolvimento da sociedade brasileira (classes socioeconômicas e rendimento). Um estudo foi realizado com 297 casos (combinação dos 11 anos com dados das variáveis dos 26 estados brasileiros mais o Distrito Federal) do período de 2001 a 2011. Com modelagem econométrica via equações de estimação generalizadas, construíram-se painel de dados por estado. Os resultados demonstram sete padrões de efeitos mútuos de que o crescimento de novas empresas é afetado pelas características da população que fornecem o contexto para seus surgimentos e as novas empresas modificam a sociedade numa relação dinâmica. A pesquisa evidenciou um ciclo de desenvolvimento na realidade brasileira, tendo como um dos principais atores a atividade empresarial.

PALAVRAS-CHAVE: atividade empresarial; novas empresas; desenvolvimento da sociedade; classe socioeconômica; rendimento.

Patrícia Sá de Roure ${ }^{1}$ Rafael Barreiros Porto ${ }^{2}$

\footnotetext{
${ }^{1}$ Mestrado em Administração pela Universidade de Brasília - UnB, Distrito Federal (Brasil)

E-mail: patriciasroure@gmail.com

${ }^{2}$ Doutorado em Ciências do Comportamento pela Universidade de Brasília - UnB, Distrito Federal (Brasil). Professor pela Universidade de Brasília - UnB, Distrito Federal (Brasil).

E-mail: rbarreirosporto@gmail.com
} 


\title{
MUTUAL EFFECT BETWEEN NEW FIRMS' GROWTH AND DEVELOPMENT OF THE BRAZILIAN SOCIETY: THE ROLE OF COMPANY ACTIVITY IN BRAZILIAN STATES
}

\begin{abstract}
Some business legal typologies in Brazil may encourage or inhibit the development of society, while the trajectory of this development can encourage or decay rates of creation of new businesses. However, these relations are poorly investigated in Brazilian literature. The research aimed to analyze the mutual effects between the creation of new firms (total and subdivided by legal types) and the development of Brazilian society (socioeconomic and income classes). A study was conducted (combination of 11 years with variable data of the 26 Brazilian states plus the Federal District) for the period 2001-2011. With econometric modeling via generalized estimating equations, the researchers built panel data with 297 cases by state. The results show seven patterns of mutual effects, signaling that firms' growth is affected by population characteristics that provide the context for their emergences and new firms change society in a dynamic relationship. The research revealed a cycle of development in the Brazilian reality, having as one of the main actors the company activity.
\end{abstract}

KEYWORDS: company activity; new firms; development of society; socioeconomic class; income. 


\section{INTRODUÇÃO}

O desenvolvimento socioeconômico de uma nação é impulsionado, dentre outros, pela criação de empresas que reflete atividades empreendedoras (Cucculelli, 2012; Gartner, 1985; Porter \& Kramer, 2011; Scarpin, Roncon, Correia, \& Hoeltgebaum, 2012). A mudança social derivada do surgimento de novas empresas pode ser analisada a partir de indicadores socioeconômicos para se compreender como esse processo ocorre (Vicenzi \& Bulgacov, 2013; Wiklund, Davidsson, Audretsch, \& Karlsson, 2011). Em específico, algumas características do nível socioeconômico de uma região - que inclui rendimento familiar, escolarização das famílias, quantidade de pessoas que trabalham e a idade do trabalhador - podem favorecer a abertura de novas empresas, e esta, por sua vez numa perspectiva dinâmica e evolucionária (Metcalfe, 2004), altera o crescimento econômico e desenvolvimento da sociedade, provocando um ciclo de influência mútua positivo ou negativo.

Em termos gerais, o crescimento da oferta de produtos e serviços oportunizado pelas novas empresas tem importante papel na economia, podendo favorecer o desenvolvimento socioeconômico por meio da competitividade empresarial (Almeida, Santos, Albuquerque, \& Ferreira, 2013; Porter \& Kramer, 2011; Reynolds \& Miller, 1992). As empresas, então, criam valor por meio da oferta de produtos e serviços vindos da mão de obra qualificada (R. P. de Brito \& L. A. L. Brito, 2012). Ao comercializar, esta mão de obra é remunerada via salários e os acionistas ou empresários se apropriam do valor remanescente. Assim, aumento no rendimento da população é esperado.

A forma como essas empresas são constituídas e as motivações para que sejam criadas podem variar conforme os tipos de empresas (oferta) e características da população, as quais compõem a demanda brasileira (Bielschowsky, 2012). Pelo lado da oferta, ao constituir uma empresa, a opção por um tipo jurídico de empresa ou outro é predominantemente influenciada por recursos disponíveis e por orientações jurídicas de direito empresarial (Coelho, 2014). Pelo lado da demanda, ao se conhecer as características da população que favorecem a criação de empresas, é possível prever em quais situações, ou diante de que cenário, é mais indicado empreender.

As classes sociais são a forma como a população é categorizada, normalmente medidas por indicadores como idade, escolaridade, ocupação e renda (Haug, 1977; Suzuki \& Patricoski, 2013). Essas características socioeconômicas da população são abordadas na literatura como sendo influenciadoras do empreendedorismo (Davidsson, 2006; Robb \& Robinson, 2014; 
Vicenzi \& Bulgacov, 2013). Além disso, Braga (2012) afirma que, ao conhecer a estrutura e o tamanho das classes sociais brasileiras, a iniciativa privada tem o conhecimento necessário para agir e lançar marcas e produtos no mercado com base na demanda existente.

Em contrapartida, é possível propor uma relação de influência dinâmica, onde as características da população influenciam os tipos de empresas criadas e estas criam novas condições para a população se desenvolver. Infelizmente, essas relações não são demonstradas com clareza na literatura, em especial a brasileira, que é formada por diversas características em cada estado brasileiro (Neri, 2011). Cada estado brasileiro tem suas peculiaridades de volume (e característica) de população e volume de tipologias de empresas diferentes (Coelho, 2014). Isso pode se dever às contingências econômicas que vive cada estado em cada época. Assim, o objetivo deste estudo é analisar os efeitos mútuos entre o crescimento de novas empresas e o desenvolvimento da sociedade brasileira em um estudo longitudinal, clareando seus efeitos incentivadores ou inibidores.

As contribuições acadêmicas do trabalho estão na ligação entre os estudos sobre criação de novas empresas com os aspectos socioeconômicos da população. As pesquisas sobre estratégias empreendedoras de empresas assumem que elas são influenciadas pelas características da demanda e que existem impactos socioeconômicos na sociedade, mas não se sabe exatamente a proporção desses efeitos. O trabalho também traz contribuições gerenciais ao alinhar os interesses de economistas e administradores quanto a identificar características da população que podem favorecer ou não a criação de um determinado tipo de empresa e que tipo de impacto na sociedade as empresas geram. Essa pesquisa visa dar a luz a esses lacunas.

\section{REFERENCIAL TEÓRICO}

\section{Atividade empresarial e a criação de novas empresas}

O processo de criação de empresas é a etapa inicial da atividade empresarial. Numa perspectiva econômica, essa atividade é articulada em quatro fatores de produção: capital, mão de obra, insumo e tecnologia que se combinam para a produção ou circulação de bens ou serviços (Coelho, 2014; Kirzner, 1978; Tigre, 2006). Em termos gerais, o principal questionamento do início da atividade empresarial é que oportunidades no ambiente que as decisões de criação da empresa são baseadas (Kirzner, 1978; Wiklund et al., 2011). Isso demanda uma análise mais minuciosa sobre o que leva empreendedores a criarem novas empresas. 
O ponto central do empreendedorismo está no surgimento de uma nova atividade empresarial, com foco no seu processo social-econômico (Wiklund et al., 2011). Logo, o empreendedorismo pode ser a busca por oportunidades de negócios, progressos tecnológicos e criação de riquezas, sendo relevante para o crescimento da economia, além de um meio para manter padrões de consumo por fomentar a economia (Scarpin et al., 2012). Isso já é documentado na literatura. Garofoli (1994) demonstra que o incentivo de um ambiente socioeconômico adequado propicia a criação de empresa e o ambiente socioeconômico desfavorável, como o aumento do desemprego, inibe sua criação (Liedholm, 2002; Storey, 1991). Segundo Davidsson (2006), a maioria dos novos empreendedores abrem empresas baseadas em oportunidades do mercado e, no cenário mais amplo, para países em desenvolvimento, as políticas que focam em explorar economias de escala, investimentos estrangeiros e melhorar a educação em negócios oferecem as condições para a criação de empresas.

Os fatores socioeconômicos que podem levar à criação de empresas passam pela idade, escolaridade, renda e condições de trabalho da população (Ferro \& Torkomian, 1988; Liedholm \& Mead, 2013; Vicenzi \& Bulgacov, 2013). Para Robb and Robinson (2014), o aumento no rendimento dos potenciais empreendedores permite que eles tenham acesso a mais linhas de crédito e faz com que estejam mais dispostos a investir em novos negócios. Assim, considerando o aumento da renda como motivadora para abrir um negócio (Kuznets, 1955), maiores taxas de novas empresas são esperadas nas regiões socioeconômicas favoráveis.

Para a criação de uma empresa, Faria e Carvalho (2011) ressaltam a importância do conhecimento dos cenários existentes de acordo com as possibilidades de variação na população. As mudanças que ocorrem no ambiente econômico se tornam para a organização indicadores das prioridades que ela deve adotar (Lins, Domingues, Del Corso, \& Costa 2012). Gartner (1985) apresenta um modelo no qual a criação de novas empresas é decorrente de quatro dimensões: individual (pessoas envolvidas no processo), organizacional (tipo de empresa criada), ambiental (cenário no qual a empresa está sendo aberta e que a influencia), e de processos (ações para abrir a empresa).

No nível individual (motivação de abrir um negócio), pode-se dizer que os traços individuais, as características sociodemográficas, as experiências anteriores, entre outros fatores são considerados antecedentes do empreendedorismo, impactando a decisão de abrir empresas (Ferro \& Torkomian, 1988; Liedholm \& Mead, 2013; Vicenzi \& Bulgacov, 2013), mas no nível da nação, a variação nas características da população pode ser a razão pela qual 
novas oportunidades são criadas no mercado, alterando sua estrutura (Faria \& Carvalho, 2011). Em específico, as mudanças socioeconômicas pelas quais a população passa moldam a demanda de acordo com as classes sociais que a compõem, com o rendimento da população e com as características familiares (Almeida et al., 2013; Fochezatto, 2011).

No contexto brasileiro, os fatores motivadores dos empreendedores são discutidos por Vicenzi e Bulgacov (2013) ao afirmar que os incentivos vão além da oportunidade ou necessidade, podendo originar de condições da família e maior possibilidade de ganhos financeiros. Os autores identificaram que a maior parte dos empreendedores são jovens até 35 anos e com alta escolaridade (formação superior/pós-graduação), e que, para abrir uma nova empresa, 42,9\% deles atuaram de acordo com a demanda no mercado. Ainda, no cenário brasileiro, Scarpin et al. (2012) encontraram que as taxas de empreendedores cresceu nos últimos anos e que, mesmo após a crise mundial de 2008, a taxa de empreendedorismo por oportunidade foi superior a $60 \%$.

Para o aumento da taxa de criação de empresas deve-se, portanto, considerar tanto as motivações pessoais dos empreendedores quanto os indicadores ambientais que se mostram como oportunidades. Gartner (1985) afirma que os empreendedores não agem indiscriminadamente, mas respondem aos seus ambientes. No Brasil, devido a peculiaridade jurídica (Sobottka et al., 2010), no momento da constituição de uma empresa é necessário que, além de decisões administrativas e estratégicas, seja realizado o registro mercantil, permitindo que ela exerça atividades econômicas de acordo com seu tipo de negócio e porte.

Para caracterizar a atuação da Junta Comercial no desenvolvimento da economia, Sobottka et al. (2010) indicam que as Juntas Comerciais são responsáveis pelo registro do ato constitutivo das empresas, pelo qual elas passam a existir e ser personalidades jurídicas, assim como definem a formatação societária. A fim de distinguir os tipos de empresas existentes e que são abertas constantemente nas Juntas Comerciais, esses órgãos utilizam a forma jurídica de empresas. Nessa classificação, as empresas dividem-se em empresário individual, sociedades empresárias (limitadas), sociedades anônimas, cooperativas e outros tipos.

Os Empresários Individuais são pessoas físicas que exercem atividade de empresário, formadas por um proprietário, que toma decisões estratégicas e tem atributos empreendedores, como buscar oportunidades (Pinheiro, 2011). Já as Sociedades Limitadas, de acordo com Tomazette (2011), exercem atividade econômica de produção ou circulação de bens ou serviços, devendo por lei efetuarem registro, com o seu capital social fruto da soma do capital dos sócios. Ainda, Tomazette (2011) descreve que as Sociedades Anônimas têm a natureza de 
sociedade de capitais, divisão do capital social em ações, responsabilidade limitada e natureza mercantil (empresarial). Por fim, as Cooperativas são associações autônomas que têm por finalidade a produção de bens ou serviços com impacto social (Campos, Martens, Resende, Carmona, \& Lima 2013).

Entende-se que alguns tipos de empresas podem se revelar mais expressivos do que outros em termos de volume em cada estado brasileiro em cada época. Essa diferenciação pode ocorrer por questões processuais, financeiras ou econômicas. Por exemplo, Empresas Individuais e Sociedades Limitadas são geralmente empresas menores e com maior incidência em qualquer estado brasileiro, enquanto Sociedades Anônimas são empresas maiores que precisam de mais capital, mais pessoas envolvidas e possuem uma maior carga de impacto na economia do país, geralmente com incidência alta em poucos estados brasileiros (Instituto Brasileiro de Geografia e Estatística [IBGE], 2013). Essas diferenças, por estado e por período temporal, dão dinamismo para compreender os impactos mútuos entre a atividade empresarial e o quanto ela é influenciada ou influenciadora do desenvolvimento da sociedade.

\section{Desenvolvimento da sociedade brasileira - classe socioeconômica e rendimento}

Parte do desenvolvimento econômico de uma nação é decorrente da criação de empresas que apresenta como resultado a geração de riquezas, de emprego e de renda na população (Tigre, 2006). Porter and Kramer (2011) afirmam que as empresas precisam de uma sociedade desenvolvida e a população precisa de negócios de sucesso. Para os autores, isso ocorre porque as empresas precisam criar demanda para seus produtos e que possam extrair lucros, assim como a sociedade precisa de empregos e de aumento de seu rendimento.

Apesar disso, existem algumas controvérsias nos resultados, quando comparados entre nações ou mesmo entre regiões do mesmo país. Alguns países com baixa taxa de empreendedorismo são mais desenvolvidos (Souza \& Lopez, 2011) e as regiões com baixo rendimento da população têm piores qualidades empreendedoras (Romero, 2011). Assim, geram-se dúvidas do possível efeito benéfico da criação de empresas (Leff, 1979). Porém, se comparado longitudinalmente na mesma região os efeitos de criação de empresa, a relação pode ser melhor investigada, demonstrando evolução e efeitos mútuos (Metcalfe, 2004). Por um lado, as características da população são fontes de oportunidades a serem exploradas pelos empresários por meio da criação de empresas e inovação (Faria \& Carvalho, 2011; Tigre, 2006). Por ouro lado, como consequência, a proliferação de empresas aumenta o desenvolvimento socioeconômico sustentável (Almeida et al., 2013; Cucculelli, 2012). 
Atualmente, é possível perceber diferenças nos padrões de consumo em cada classe social brasileira, as quais têm adotado novas formas de se relacionar com o mercado. A fim de caracterizar as classes sociais, Piff, Kraus, Côté, Cheng, and Keltner (2010) identificaram que as diferenças entre as classes mais baixas e as mais altas são principalmente que as primeiras têm menos recursos econômicos, oportunidades de educação, acesso a instituições sociais e que estão em uma posição subordinada em relação às outras classes. Baseadas na ocupação, escolaridade e riqueza, as classes sociais são contextos sociais que influenciam como os indivíduos se comportam. Propostas similares são compartilhadas por Suzuki and Patricoski (2013). Fochezatto (2011) complementa ao relatar que o perfil da distribuição de renda na população gera diferentes resultados na estrutura produtiva da economia, e Haug (1977) afirma que a ocupação do indivíduo deve ser combinada com outros indicadores das classes porque, em geral, a renda varia de acordo com a ocupação.

Portanto, as classes sociais brasileiras, bem como, seu rendimento, podem fornecer insumos para compreender como os empresários atendem à demanda agregada e, numa perspectiva dinâmica, como as atividades empresariais empreendidas por eles têm alterado o desenvolvimento da sociedade brasileira. Especificamente, as novas empresas criadas podem estimular o aumento do rendimento e do volume das classes médias ou altas e simultaneamente reduzir o volume das classes baixas. Caso estejam estimulando essa situação, há indícios de desenvolvimento. Caso esteja aumentando a classe baixa e diminuindo o rendimento e o volume da classe média ou alta há indício de retrocesso. A presente pesquisa visa demonstrar o que ocorre na realidade brasileira.

\section{MÉTODO}

Foi realizada uma pesquisa ex post facto (Kerlinger, 1973), visando identificar os efeitos temporais entre as variáveis estudadas após terem ocorrido. Ela é usada para explicar consequências baseadas em antecedentes que o pesquisador não pôde manipular. De natureza correlacional, priorizou-se utilização de modelagem econométrica para evidenciar as influências mútuas das variáveis.

A pesquisa* se divide em dois modelos. O primeiro modelo (Figura 1) tem o desenvolvimento da sociedade brasileira - composto pela quantidade de família das classes sociais (baseado em renda, idade, escolaridade) e rendimento em reais da população economicamente ativa (PEA) - como variáveis independentes (VIs) e a criação de empresas - 
total de novas empresas e quantidade de novas empresas em cada tipo jurídico (empresas individuais, sociedades limitadas, sociedades anônimas, cooperativas e outras empresas) como variáveis dependentes (VDs).

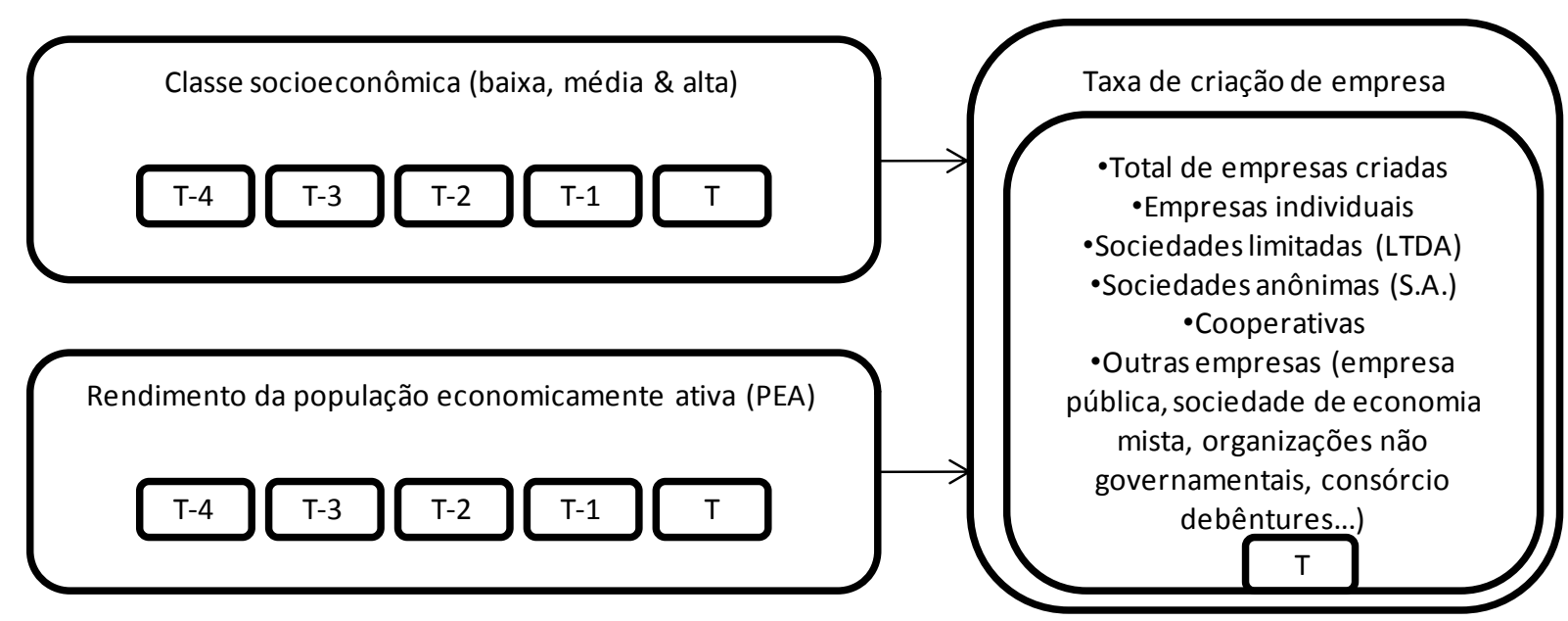

Figura 1. Modelo de influência do desenvolvimento da sociedade sobre a criação de novas empresas

O segundo modelo é a retroalimentação do primeiro (Figura 2). Assim, ele possui como VIs o total de novas empresas criadas com seus tipos jurídicos e o desenvolvimento da sociedade brasileira, constituído pelas classes e o rendimento da população, como VDs. Considera-se que as VIs possuem uma possível influência defasada nas VDs e por isso optouse por incluir tanto o efeito corrente anual (T) quanto o defasado em até 4 anos (T-4) nas equações estimadas. Isso permite que se analise um acontecimento anterior, mas que gera consequências futuras no consolidado por 5 anos. Optou-se por considerar como variáveis de controle o ano, o estado e o crescimento populacional por estado. Assim, independente do ano, dos acontecimentos que variam de um estado para outro, ou do crescimento da população, os efeitos encontrados são mais fidedignos, sem a interferência da variação dessas variáveis. 


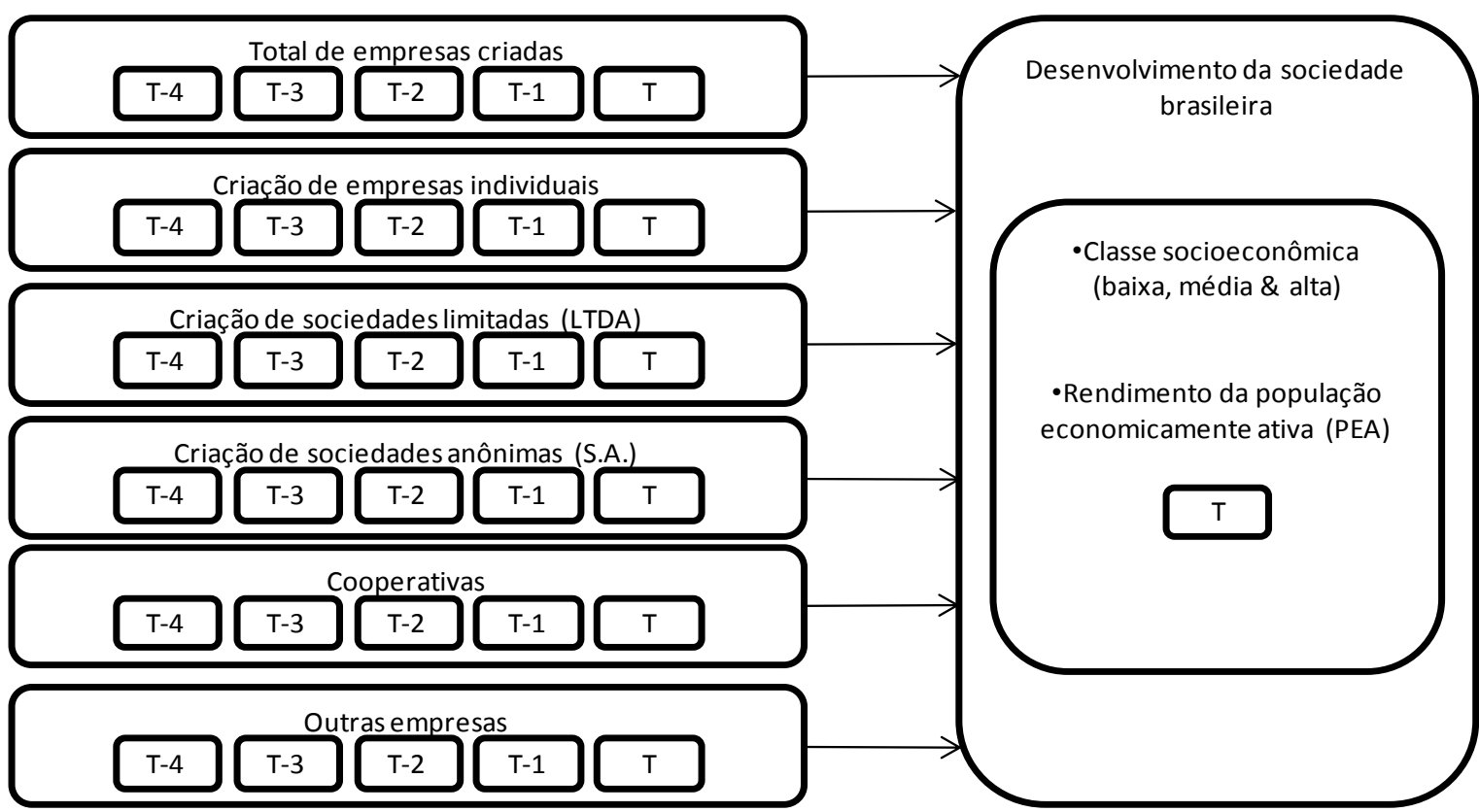

Figura 2. Modelo de influência da criação de novas empresas sobre o desenvolvimento da sociedade

No que se refere ao horizonte temporal, foi utilizado os anos no período de 2001 a 2011. Dessa forma, foi possível identificar os efeitos de uma variável sobre a outra ao longo do tempo, permitindo estabelecer uma relação de antecedência e consequência. O período foi delimitado por ser o prazo disponível para consulta das variáveis junto aos órgãos responsáveis. Os dados utilizados na pesquisa são secundários e foram coletados nos sítios virtuais do Instituto Brasileiro de Geografia e Estatística, das Juntas Comerciais e do Departamento Nacional de Registro do Comércio.

O tamanho da amostra geral do painel foi na ordem de 297 casos (combinação dos 11 anos com dados das variáveis dos 26 estados brasileiros mais o Distrito Federal) para as análises que contenham o total de empresas criadas. Para as análises que contenham os tipos jurídicos como variáveis, a amostra foi menor (variou de 78 a 165 casos). Isso porque nem todos os estados forneceram todos os dados de cada tipologia em cada período. O teste de poder amostral para a menor amostra foi na ordem de $80,4 \%$. Ainda assim, nesta menor amostra, ele é muito bom para evitar o Erro Tipo 2.

A taxa média de criação de empresas foi na ordem de 19.305,2 (desvio padrão $=30.599$, 5) empresas ao ano em todo o Brasil, sendo 9.758,3 (desvio padrão $=9.613,0$ ) empresas individuais, 11.510,2 (desvio padrão $=9.704,7$ ) LTDA, 144,2 (desvio padrão = 214,3) S.A., $70,9$ (desvio padrão $=59,7)$ cooperativas e 71,6 (desvio padrão $=460,0)$ outras empresas. A 
média do rendimento médio em cada estado foi igual a $\mathrm{R} \$ 512,75$ (desvio padrão $=245,95$ ) no período analisado.

Para que as classes sociais pudessem ser medidas como parte do desenvolvimento da sociedade brasileira, foram utilizados os dados de famílias por renda familiar, idade e escolaridade da população economicamente ativa (PEA). Para a categorização das classes sociais de classe baixa, média e alta, as variáveis foram determinadas após análise de componentes principais Promax com normalização Kaiser da idade e escolaridade da PEA e da quantidade de famílias por renda familiar disponibilizados pela pesquisa de Amostra por Domicílio do IBGE (2013). Essa análise indicou a fatorabilidade da matriz de correlações $(\mathrm{KMO}=0,89$, Teste de Esfericidade Barlett $=$ Qui-quadrado 25.916,82; $\mathrm{p}<0,01)$. Obteve-se duas dimensões como pode ser visto na Tabela 1.

Com base nas características de cada um dos fatores, optou-se por denominá-los de classe baixa (Fator 1) e classe média \& alta (Fator 2) por refletirem a classificação socioeconômica. Essa análise não evidenciou separação entre a classe média da classe alta e por isso elas foram condensadas em uma só dimensão. A classe baixa foi formada por famílias com renda até 2 salários mínimos (s.m.), pela escolaridade variando de sem instrução a 3 anos e com maior volume de pessoas do PEA que trabalham em idade de 10 a 14 anos. Por sua vez, a classe média $\&$ alta incluiu famílias com renda de 3 a mais de 20 s.m., com escolaridade de 4 a 15 anos ou mais e maior volume da população economicamente ativa em idade acima de 15 anos.

Com o fim de padronizar os dados, todas as variáveis foram divididas pela média da variável durante todo o período e transformadas em logaritmo, fazendo com que elas tivessem valores relativos à média em pontos percentuais (elasticidade). Nesse caso, a média passou a ser 0 . Se acima (abaixo) de 0 o valor está acima (abaixo) da média.

Para o tratamento e análise dos dados, não houve problemas de multicolinearidade (VIF < 10) e de homocedasticidade (teste White LM p > 0,05). Porém, as variáveis dependentes não eram normais pelo teste Kolmogorov-Smirnov $(\mathrm{p}<0,05)$ e de acordo com o teste de DurbinWatson, há autocorrelação da variável dependente. Isso demanda uma técnica estatística semiparamétrica (Ballinger, 2004). Por atender a esses pressupostos, as Equações de Estimação Generalizadas (GEE) foram adequadas para a análise da presente pesquisa longitudinal. As análises foram realizadas testando-se a matriz de correlação de trabalho, a qual apresentou melhores resultados para a não estruturada, a indicada quando não há um padrão de autocorrelação das variáveis dependentes medido pelo Quasi-likelihood Information Criteria (QIC - indicador de ajuste dos dados). 
Tabela 1. Cargas Fatoriais, Variância Explicada, Akpha de Cronbach e Indicadores das Classes

\begin{tabular}{|c|c|c|}
\hline & \multicolumn{2}{|c|}{ FATORES } \\
\hline & 1 & 2 \\
\hline Variância explicada & 18,38 & 12,75 \\
\hline Número de itens & 18 & 4 \\
\hline Alphas de Cronbach & 0,98 & 0,91 \\
\hline Pop. Economicamente Ativa por escolaridade de 15 ou mais anos & 1,118 & \\
\hline Famílias por renda familiar de mais de 5 a 10 salários mínimos & 1,108 & \\
\hline Famílias por renda familiar de mais de 10 a 20 salários mínimos & 1,107 & \\
\hline Famílias por renda familiar de mais de 20 salários mínimos & 1,060 & \\
\hline Famílias por renda familiar de mais de 3 a 5 salários mínimos & 1,051 & \\
\hline Pop. Economicamente Ativa por escolaridade de 11 a 14 anos & 1,024 & \\
\hline Pop. Economicamente Ativa por escolaridade de 8 a 10 anos & 0,974 & \\
\hline Pop. Economicamente Ativa por idade de 40 a 49 anos & 0,944 & \\
\hline Pop. Economicamente Ativa por idade de 50 a 59 anos & 0,917 & \\
\hline Pop. Economicamente Ativa por idade de 30 a 39 anos & 0,910 & \\
\hline Pop. Economicamente Ativa por idade de 25 a 29 anos & 0,892 & \\
\hline Famílias por renda familiar de mais de 2 a 3 salários mínimos & 0,873 & \\
\hline Pop. Economicamente Ativa por idade de 20 a 24 anos & 0,852 & \\
\hline Pop. Economicamente Ativa por escolaridade de 4 a 7 anos & 0,754 & \\
\hline Pop. Economicamente Ativa por idade de 15 a 19 anos & 0,752 & \\
\hline Pop. Economicamente Ativa por idade de 60 anos ou mais & 0,695 & \\
\hline Famílias por renda familiar - sem rendimento & 0,645 & \\
\hline Famílias por renda familiar de mais de 1 a 2 salários mínimos & 0,569 & \\
\hline $\begin{array}{l}\text { Pop. Economicamente Ativa por escolaridade - sem instrução a } \\
\text { menos de } 1 \text { ano }\end{array}$ & & 1,107 \\
\hline Pop. Economicamente Ativa por idade de 10 a 14 anos & & 1,080 \\
\hline Famílias por renda familiar até 1 salário mínimo & & 1,008 \\
\hline Pop. Economicamente Ativa por escolaridade de 1 a 3 anos & & 0,798 \\
\hline
\end{tabular}

Nota. Método de extração: Análise dos componentes principais. Método de rotação: Promax com normalização Kaiser.

\section{RESULTADOS}

O comportamento descritivo das VIs e VDs ao longo do tempo para alguns estados brasileiros é visto na Figura 3. Quando aumenta a quantidade de famílias de classe média \& alta e diminui a quantidade de famílias de classe baixa, geralmente aumenta o total de empresas criadas, mas aparentemente com efeitos defasados. O rendimento PEA também contribui com 
a criação de empresa, mas parece ter um efeito dúbio. Quando ele cresce, a taxa de empresas também cresce, mas não de forma regular.

O comportamento dessas variáveis para os estados do Acre e Paraíba têm maior instabilidade em pontos percentuais do que os estados de Paraná e São Paulo, o que indica que a variação de uma variável pode de fato estar influenciando o comportamento de outra variável. O estado de Goiás se comporta parecido com a média nacional. Contudo, além das empresas criadas poderem ter grau de variação diferente em cada tipologia jurídica, há efeito de variáveis perturbadoras (confounding variables) que podem distorcer esses resultados e devem ser controladas. Igualmente, o efeito (elasticidade corrente e defasada) entre elas não é verificável com exatidão pelos gráficos. Por isso, análises provenientes da GEE podem demonstra $\mathrm{r}$ melhor esses efeitos.
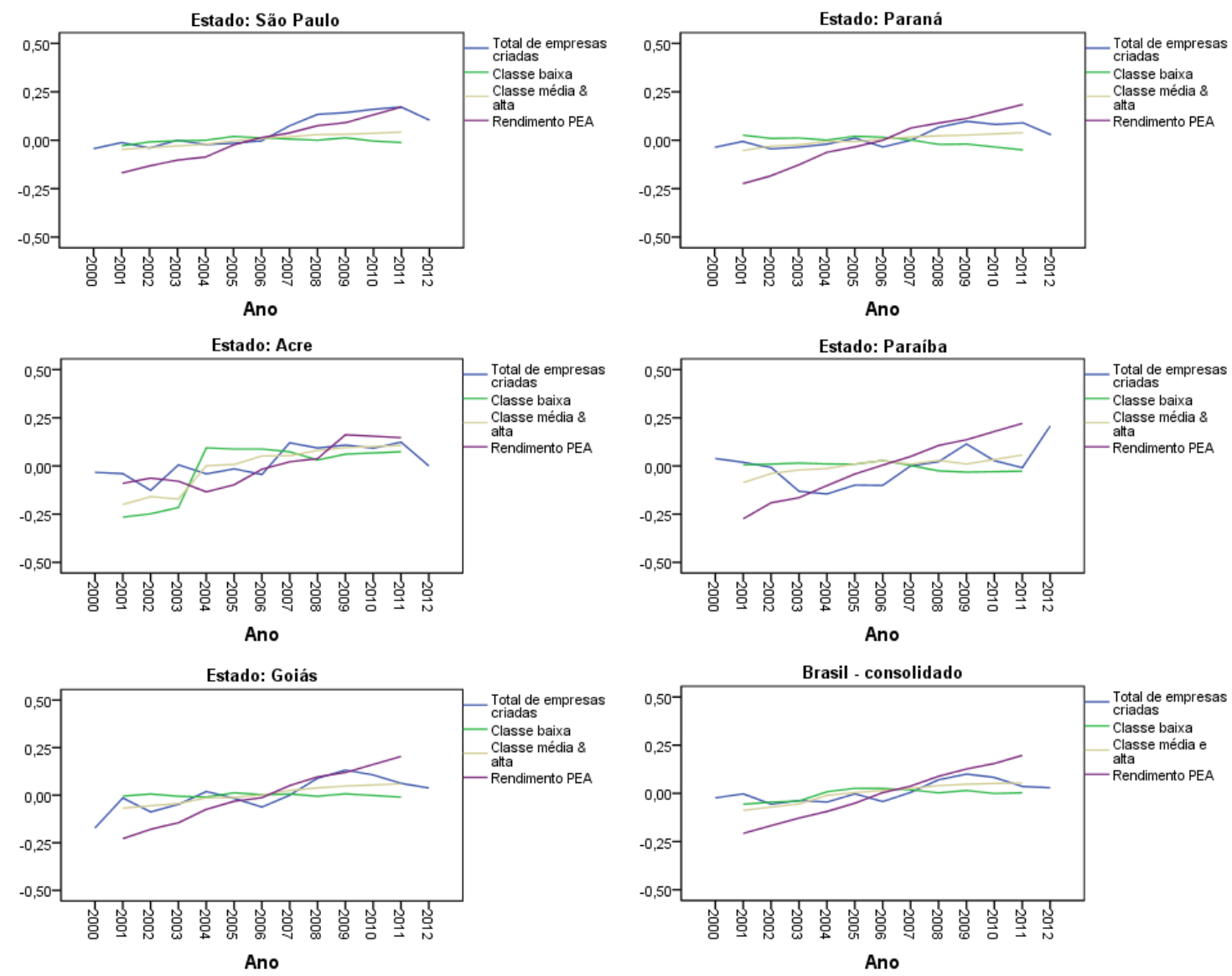

Figura 3. Gráficos do comportamento das VIs e VDs em alguns estados brasileiros

Efeito do desenvolvimento da sociedade na criação de novas empresas (Modelo 1)

A relação temporal do efeito do desenvolvimento da sociedade brasileira em cada estado sobre a criação de empresa demonstrou ter bons ajustes [(o QICC variou entre 92,23 (VD: total de empresas criadas) a 66,41 (VD: cooperativas criadas), com valor de referência (modelo 
apenas com o intercepto) igual a 198,02]. Com efeito controlado do ano, estado e crescimento populacional, essa relação apresentou também boa variância explicada $\left(\mathrm{R}^{2}=65,9 \%\right.$ para o total de empresas criadas; $\mathrm{R}^{2}=91,4 \%$; para empresas individuais; $\mathrm{R}^{2}=88,1 \%$ para LTDA; $\mathrm{R}^{2}=$ $64,9 \%$ para S.A.; $\mathrm{R}^{2}=58,8 \%$ para cooperativas e $\mathrm{R}^{2}=92,3 \%$ para outras empresas).

A Tabela 2 demonstra os efeitos em cada ano - efeito corrente (T) e efeitos defasados (T1 a T-4) - de cada variável independente (rendimento PEA, classe baixa e classe média \& alta) para cada tipologia jurídica de empresa (variáveis dependentes do Modelo 1) em cada estado. Observa-se que quanto mais famílias existem de classe baixa, menores as taxas em pontos percentuais de criação de empresa. Isso ocorre para todos os tipos jurídicos de empresa e para o total de empresas criadas. Esses efeitos da classe baixa ocorrem em até dois anos (T-2) após o crescimento da quantidade de família. É um contexto socioeconômico que inibe novas empresas.

Também observa-se que quanto mais famílias existem de classe média \& alta, maiores as taxas em pontos percentuais de criação de empresa. Isso ocorre para todos os tipos jurídicos de empresa e para o total de empresas criadas, tendo um possível efeito negativo em T-2 para LTDA, mas esse efeito é menor do que o efeito positivo em T-1 para essas mesmas empresas. É um contexto socioeconômico que favorece o surgimento de novas empresas.

Além disso, um padrão de efeito foi encontrado tendo o rendimento como variável independente: quanto menor o rendimento da população economicamente ativa, maior a taxa de criação de empresas para todas as tipologias jurídicas. Esse efeito é visível tanto a curto prazo (T), quanto a médio e longo prazo (T-1 a T-4). Em especial, quanto mais declinante o rendimento, mais cooperativas e outras empresas são criadas. Outras empresas também são criadas, mas com efeitos menores. O efeito mais tardio é visto para Sociedades Anônimas e sociedades Limitadas. Estima-se que se o rendimento é baixo, demora-se 4 anos para haver aumentos nas Sociedades Anônimas e 3 anos para haver aumentos nas Limitadas. 
Tabela 2. Efeito do Desenvolvimento da Sociedade Brasileira na Criação de Novas Empresas

\begin{tabular}{|c|c|c|c|c|c|c|c|c|c|c|c|c|c|}
\hline \multirow[b]{2}{*}{ VI } & \multirow[b]{2}{*}{ Período } & \multicolumn{2}{|c|}{$\begin{array}{l}\text { VD: total de } \\
\text { empresas }\end{array}$} & \multicolumn{2}{|c|}{$\begin{array}{l}\text { VD: empresas } \\
\text { individuais }\end{array}$} & \multicolumn{2}{|c|}{ VD: LTDA } & \multicolumn{2}{|c|}{ VD: S.A. } & \multicolumn{2}{|c|}{$\begin{array}{c}\text { VD: } \\
\text { Cooperativas }\end{array}$} & \multicolumn{2}{|c|}{$\begin{array}{l}\text { VD: Outras } \\
\text { empresas }\end{array}$} \\
\hline & & Estimat. & $\begin{array}{l}\text { Erro } \\
\text { Pad. }\end{array}$ & Estimat. & $\begin{array}{l}\text { Erro } \\
\text { Pad. }\end{array}$ & Estimat. & $\begin{array}{l}\text { Erro } \\
\text { Pad. }\end{array}$ & Estimat. & $\begin{array}{l}\text { Erro } \\
\text { Pad. }\end{array}$ & Es timat. & $\begin{array}{l}\text { Erro } \\
\text { Pad. }\end{array}$ & Estimat. & $\begin{array}{l}\text { Erro } \\
\text { Pad. }\end{array}$ \\
\hline \multirow{5}{*}{$\begin{array}{l}\text { Rendimento } \\
\text { PEA }\end{array}$} & $\mathrm{T}$ & $-0,40^{*}$ & 0,17 & $-0,88 *$ & 0,16 & & & & & $-2,21 *$ & 0,92 & & \\
\hline & $\mathrm{T}-1$ & $-0,47^{*}$ & 0,22 & $-0,53 *$ & 0,19 & & & & & $-2,33^{*}$ & 0,68 & & \\
\hline & $\mathrm{T}-2$ & $-0,48^{*}$ & 0,22 & & & & & & & $-1,79^{*}$ & 0,87 & $-2,66^{*}$ & 1,26 \\
\hline & $\mathrm{T}-3$ & $-0,72 *$ & 0,34 & & & $-0,60^{*}$ & 0,12 & & & $-3,66^{*}$ & 1 & & \\
\hline & $\mathrm{T}-4$ & $-0,77^{*}$ & 0,28 & & & & & $-2,56^{*}$ & 0,96 & & & $-3,69^{*}$ & 1,59 \\
\hline \multirow{5}{*}{$\begin{array}{l}\text { Classe } \\
\text { média \& alta }\end{array}$} & $\mathrm{T}$ & & & & & & & & & $3,62^{*}$ & 1,53 & & \\
\hline & $\mathrm{T}-1$ & & & $1,05^{*}$ & 0,25 & $0,73^{*}$ & 0,29 & & & $3,39 *$ & 1,2 & & \\
\hline & $\mathrm{T}-2$ & & & & & $-0,50^{*}$ & 0,26 & $2,10^{*}$ & 0,72 & $4,60 *$ & 1,18 & $6,02 *$ & 1,42 \\
\hline & $\mathrm{T}-3$ & $0,65^{*}$ & 0,35 & & & & & & & $3,35^{*}$ & 1,74 & $5,87^{*}$ & 2,15 \\
\hline & $\mathrm{T}-4$ & & & & & & & & & & & & \\
\hline \multirow{5}{*}{ Classe baixa } & $\mathrm{T}$ & & & & & & & $-2,25 *$ & 1,1 & $-2,14^{*}$ & 0,76 & & \\
\hline & $\mathrm{T}-1$ & $-0,87^{*}$ & 0,34 & & & $-0,42 * *$ & 0,17 & & & & & $-4,72 *$ & 1,6 \\
\hline & $\mathrm{T}-2$ & & & $-0,50 *$ & 0,13 & & & $-2,01 *$ & 0,63 & $-3,01 *$ & 0,74 & $-2,74^{*}$ & 0,88 \\
\hline & $\mathrm{T}-3$ & & & & & & & & & & & & \\
\hline & $\mathrm{T}-4$ & & & & & & & & & & & & \\
\hline
\end{tabular}

Nota. Apenas resultados significativos são mostrados: ${ }^{*} \mathrm{p} \leq 0,01 ; * * \mathrm{p} \leq 0,05$.

Pela Figura 4, observa-se o efeito agregado de 5 anos [soma das estimativas (B) de T-4 até T] de cada variável independente sobre cada tipologia jurídica de criação de empresa. Percebe-se um padrão de efeito de todas as variáveis independentes (contextos socioeconômicos) sobre a criação de empresas. Quanto mais famílias existem de classe baixa e maior o rendimento PEA, menores as taxas de criação de empresa. Quanto mais famílias existem de classe média \& alta, maiores as taxas de criação de empresa. Assim, a forma como a sociedade caminha nesses quesitos influencia a capacidade das atividades empresariais da nação, mesmo considerando a diversidade de realidade socioeconômica em cada estado, choques anuais na economia e crescimento da população.

A relação temporal do efeito da taxa de criação de empresa no desenvolvimento da sociedade brasileira em cada estado demonstrou ter igualmente bons ajustes [(o QICC variou entre 46,05 (VD: rendimento PEA) a 76,01 (VD: classe baixa), com valor de referência (modelo apenas com o intercepto) igual a 156,83]. Com efeito controlado do ano, estado e crescimento populacional, essa relação apresentou também boa variância explicada $\left(\mathrm{R}^{2}=99,4 \%\right.$ para o rendimento PEA; $\mathrm{R}^{2}=95,1 \%$; para a classe baixa $\mathrm{R}^{2}=91,5 \%$ para a classe média \& alta). 


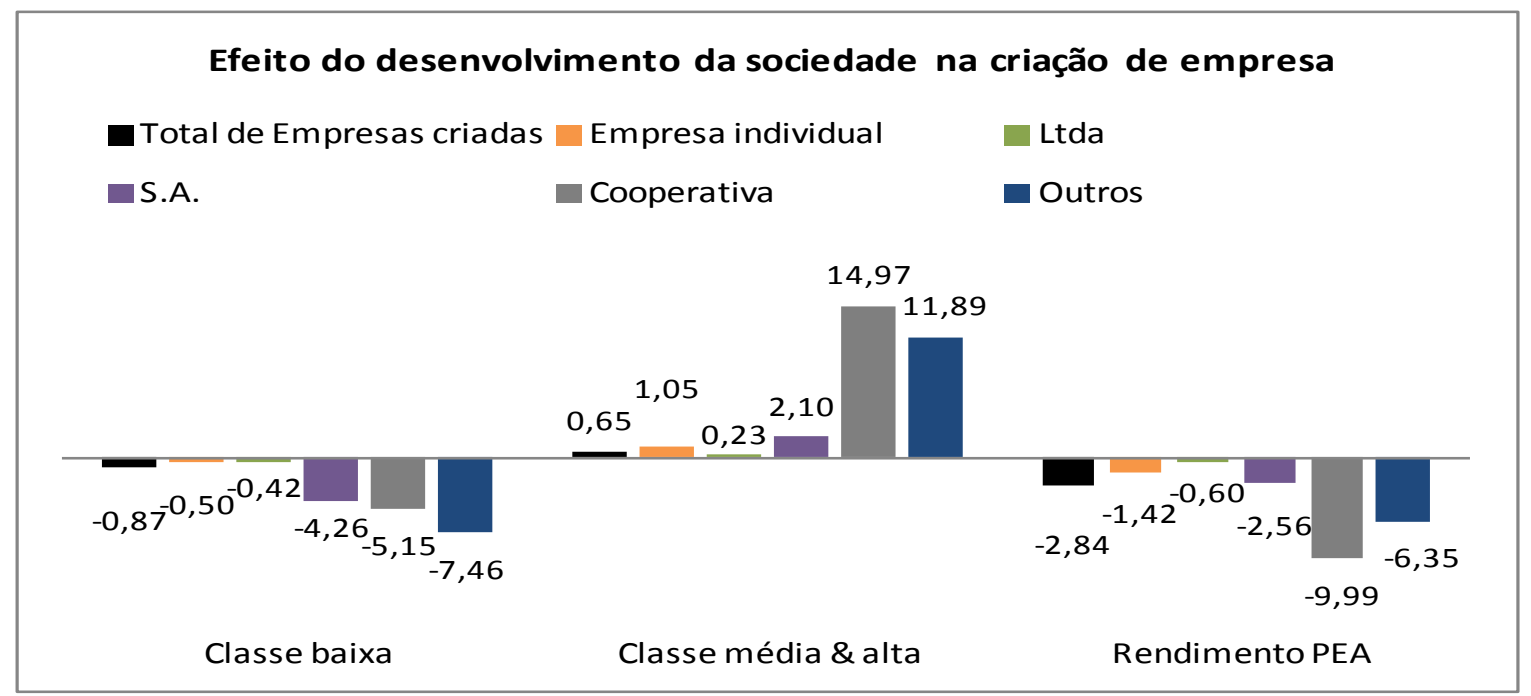

Nota. Apenas os resultados significativos estão apresentados.

Figura 4. Efeitos do desenvolvimento da sociedade brasileira sobre a criação de novas empresas

\section{Efeito da criação de novas empresas no desenvolvimento da sociedade (Modelo 2)}

A Tabela 3 demonstra os efeitos em cada ano - efeito corrente (T) e efeitos defasados (T1 a T-4) - de cada variável independente (total de empresas criadas, empresas individuais criadas, LTDA, S.A., cooperativas e outras empresas) para cada dimensão do desenvolvimento socioeconômico da sociedade (variáveis dependentes do Modelo 2) em cada estado. Observase que quanto mais empresas são criadas, em especial LTDA e S.A., maior em pontos percentuais o rendimento da PEA. Isso ocorre para todos os tipos jurídicos de empresa e para o total de empresas criadas, porém para alguns (empresas individuais e cooperativas) os efeitos benéficos em um ano são reduzidos pelos efeitos negativos em outro ano. Os efeitos podem ser visíveis tanto a curto prazo (T) quanto a longo prazo (até T-4). A criação de empresa é uma iniciativa que permite criar um novo contexto socioeconômico e seu aumento demonstra desenvolvimento socioeconômico.

Já tendo como variável dependente a classe baixa, os resultados são intrigantes. A curto prazo (T), as iniciativas empresariais de todas as empresas parecem aumentar a classe baixa, mas se considerado o efeito de longo prazo (até T-4), observa-se um declínio na quantidade de classe baixa. Em termos gerais, observa-se que quanto mais iniciativas empresariais em cada tipologia, menor em pontos percentuais a quantidade de família de classe baixa, em especial para LTDA e exceção para S.A. 
Tabela 3. Impacto da Criação de Novas Empresas no Desenvolvimento da Sociedade Brasileira

\begin{tabular}{|c|c|c|c|c|c|c|c|}
\hline \multirow[b]{2}{*}{ VI } & \multirow[b]{2}{*}{ Período } & \multicolumn{2}{|c|}{ VD: rendimento PEA } & \multicolumn{2}{|c|}{ VD: classe baixa } & \multicolumn{2}{|c|}{ VD: classe média \& alta } \\
\hline & & Estimat. & Erro Pad. & Estimat. & Erro Pad. & Estimat. & Erro Pad. \\
\hline \multirow{5}{*}{$\begin{array}{l}\text { Total de } \\
\text { empresas } \\
\text { criadas }\end{array}$} & $\mathrm{T}$ & $0,12 * *$ & 0,06 & $0,14^{*}$ & 0,01 & $0,14^{*}$ & 0,00 \\
\hline & $\mathrm{T}-1$ & & & $-0,37 *$ & 0,04 & $0,09 *$ & 0,00 \\
\hline & $\mathrm{T}-2$ & & & & & $0,01 * *$ & 0,00 \\
\hline & $\mathrm{T}-3$ & $0,22 *$ & 0,04 & & & $0,12 *$ & 0,00 \\
\hline & $\mathrm{T}-4$ & & & $-0,23 *$ & 0,01 & $-0,05 * *$ & 0,00 \\
\hline \multirow{5}{*}{$\begin{array}{l}\text { Empresas } \\
\text { individuais }\end{array}$} & $\mathrm{T}$ & $-0,12 * *$ & 0,05 & $-0,11 *$ & 0,04 & & \\
\hline & $\mathrm{T}-1$ & $0,17^{*}$ & 0,07 & & & $0,06^{*}$ & 0,02 \\
\hline & $\mathrm{T}-2$ & & & $0,08^{*}$ & 0,03 & & \\
\hline & $\mathrm{T}-3$ & & & & & & \\
\hline & $\mathrm{T}-4$ & & & & & & \\
\hline \multirow{5}{*}{$\begin{array}{l}\text { Empresas de } \\
\text { sociedades } \\
\text { limitadas } \\
\text { (LTDA) }\end{array}$} & $\mathrm{T}$ & $0,14 *$ & 0,07 & & & & \\
\hline & $\mathrm{T}-1$ & & & $-0,16^{*}$ & 0,07 & & \\
\hline & $\mathrm{T}-2$ & & & $-0,14^{*}$ & 0,07 & & \\
\hline & $\mathrm{T}-3$ & & & $-0,23 *$ & 0,04 & $0,06^{*}$ & 0,02 \\
\hline & $\mathrm{T}-4$ & & & & & & \\
\hline \multirow{5}{*}{$\begin{array}{l}\text { Empresas de } \\
\text { sociedades } \\
\text { anônimas } \\
\text { (S.A.) }\end{array}$} & $\mathrm{T}$ & & & & & & \\
\hline & $\mathrm{T}-1$ & $0,08 *$ & 0,02 & $0,05 * *$ & 0,02 & & \\
\hline & $\mathrm{T}-2$ & $0,05^{*}$ & 0,01 & & & & \\
\hline & $\mathrm{T}-3$ & & & & & $0,02 * *$ & 0,01 \\
\hline & $\mathrm{T}-4$ & & & $-0,04 * *$ & 0,01 & & \\
\hline \multirow{5}{*}{$\begin{array}{l}\text { Empresas de } \\
\text { cooperativas }\end{array}$} & $\mathrm{T}$ & & & & & & \\
\hline & $\mathrm{T}-1$ & & & $-0,06 * *$ & 0,02 & & \\
\hline & $\mathrm{T}-2$ & $0,02 * *$ & 0,01 & & & $0,01 * *$ & 0,01 \\
\hline & $\mathrm{T}-3$ & & & & & & \\
\hline & $\mathrm{T}-4$ & $-0,02 * *$ & 0,01 & & & & \\
\hline \multirow{5}{*}{$\begin{array}{l}\text { Outras } \\
\text { empresas }\end{array}$} & $\mathrm{T}$ & & & $-0,05^{* *}$ & 0,01 & & \\
\hline & $\mathrm{T}-1$ & & & & & $0,01 * *$ & 0,00 \\
\hline & $\mathrm{T}-2$ & & & $-0,02 * *$ & 0,00 & $0,01 * *$ & 0,00 \\
\hline & $\mathrm{T}-3$ & & & $-0,02 * *$ & 0,01 & & \\
\hline & $\mathrm{T}-4$ & $0,01 * *$ & 0,00 & & & $-0,01 * *$ & 0,00 \\
\hline
\end{tabular}

Nota. Apenas resultados significativos são mostrados: ${ }^{*} \mathrm{p} \leq 0,01 ; * * \mathrm{p} \leq 0,05$.

A curto e médio prazo ( $\mathrm{T}$ até $\mathrm{T}-3$ ) os efeitos das iniciativas empresariais aumentam a quantidade de famílias de classe média \& alta, mas a longo prazo (T-4) os efeitos começam a se reverter para os estados brasileiros. Como não foi testado períodos anteriores a T-4, esse achado precisa ser mencionado com cautela. Os efeitos positivos das iniciativas empresariais sobre a classe média \& alta são mais visíveis para empresas individuais e LTDA, mas geralmente a médio prazo.

Pela Figura 5, observa-se o efeito agregado de 5 anos [soma das estimativas (B) de T-4 até T] de cada variável independente sobre cada dimensão do desenvolvimento da sociedade brasileira. Percebe-se também um padrão de efeito de todas as variáveis independentes (novas 
iniciativas empresariais) sobre o desenvolvimento socioeconômico. Quanto mais empresas são criadas, maior a quantidade de família de classe média $\&$ alta e o rendimento da população economicamente ativa. Elas geralmente incentivam a redução da quantidade de família de classe baixa, com exceção das S.A. Assim, ao se criar empresas parece ser um meio de desenvolvimento socioeconômico da nação, mesmo considerando a diversidade de realidade socioeconômica em cada estado, choques anuais na economia e crescimento da população.

Nota. Apenas os resultados significativos estão apresentados.

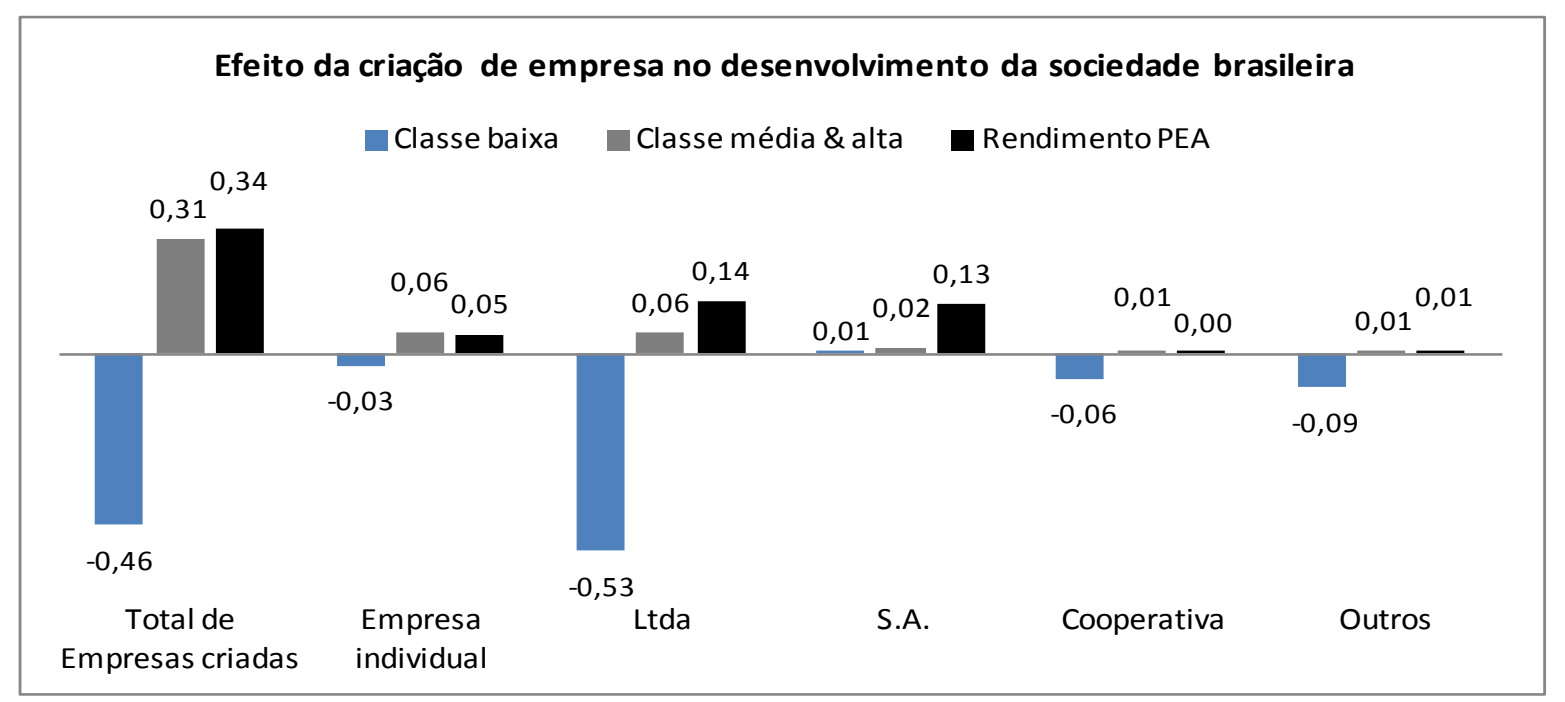

Figura 5. Efeitos da criação de novas empresas sobre o desenvolvimento da sociedade brasileira

\section{DISCUSSÃo}

Os resultados demonstram que há relações dinâmicas entre a criação de novas empresa e o desenvolvimento da sociedade brasileira. Assim, revela-se que novas empresas são criadas para atenderem a oportunidades no mercado no sentido macroeconômico (Davidsson, 2006), um dado que no nível individual (motivações de empreendedores para abrir uma empresa) é relatado (Bielschowsky, 2012; Vicenzi \& Bulgacov, 2013), incluindo para micro e pequenas empresas (Ferro \& Torkomian, 1988; Liedholm \& Mead, 2013). O estudo ratifica também que a criação de novas empresas influencia positivamente o desenvolvimento da sociedade (Cucculelli, 2012). Portanto, o fenômeno de criação de empresa, não parece estar apenas correlacionado com o desenvolvimento ou características da população (Souza \& Lopez, 2011), mas demonstra ser tanto causa quanto consequência do desenvolvimento socioeconômico, sendo um processo evolucionário (Metcalfe, 2004). 
Entretanto, seus efeitos em pontos percentuais geralmente são maiores enquanto consequência do que como causa do desenvolvimento, o que é evidenciado na presente pesquisa pelas elasticidades maiores encontradas no primeiro modelo do que no segundo modelo. Esse achado esclarece algumas incongruências na literatura estrangeira sobre o ambiente socioeconômico estimulador da criação de empresas, mas a demanda não tendo relação com a criação de empresa (Garofoli, 1994). Há determinadas características prévias da sociedade que propiciam o surgimento de empresas (ex: rendimento baixo e muito volume de classe baixa e alto volume de classe média e alta) e o contrário dessas características, o desestimulam. Caso seja estimulado, os empresários, ou sócios de empresas, se motivam em larga escala (dados agregados) a criarem empresas para atender essa demanda, se tiverem capital, recursos e mão de obra capacitada para tal (Coelho, 2014; Kirzner, 1978; Tigre, 2006). Posteriormente, essas empresas alteram as características da sociedade (Metcalfe, 2004) e por consequência, a demanda, tornando-a dinâmica.

Nas situações em que o surgimento de novas empresa é consequência do desenvolvimento, sociedades que incentivam maior quantidade de famílias de classe média e alta e desestimulam a proliferação de classe baixa criam condições favoráveis ao seu surgimento. Essa situação, aliado ao papel do fornecimento de crédito (Robb \& Robinson, 2014), auxilia o surgimento de empresa (Garofoli, 1994; Haug, 1977; Stinchcombe, 2000; Suzuki \& Patricoski, 2013). Um resultado intrigante na realidade brasileira foi encontrado principalmente aplicado para cooperativas e outras empresas (estatais, organizações não governamentais, empresas de economia mista, etc). Quanto menos famílias na classe baixa e mais famílias na classe média e alta, maior a incidência de cooperativas e outras empresas. Geralmente elas surgem devido ao papel social (Campos et al., 2013), em especial por inserirem pessoas em situação de desvantagem por condição física, mental ou social no mercado de trabalho (Martins, 2009). Mas se há muitas famílias vulneráveis numa região, sem haver famílias mais favorecidas, não parece ser um ambiente propicio para sua criação. Assim, seu papel parece ser fomentado com a existência de classe média e alta que pretende "ajudar" as classes menos favorecidas, criando-as. Todavia, as empresas tipicamente formadas no Brasil em termos de incidência (LTDA, empresas individuais e S.A.) também recebem influências das características pré-existentes da população a curto e médio prazo (até T-3), tendo o mesmo comportamento das cooperativas, mas em menor grau.

Ademais, o baixo rendimento médio de uma região, como possível decorrência de maiores taxas de desemprego (Liedholm, 2002; Storey, 1991), é um padrão incentivador da 
criação de empresas em todos os estados brasileiros, sendo demonstrado na presente pesquisa. Como algumas pesquisas utilizam corte transversal, comparando entre nações ou regiões, seus resultados parecem ser controversos, relatando que regiões de menor rendimento tem piores qualidades empreendedoras (Romero, 2011) ou que atividades empreendedoras não levam ao desenvolvimento econômico por si só (Leff, 1979). Mas ao adotar estudos longitudinais (Liedholm \& Mead, 2013), controlando efeitos de variáveis perturbadoras, evidencia-se essa relação clássica (Kuznets, 1955) de maneira mais lógica. Assim, a população com rendimento baixo é um contexto "causador" da abertura de novas empresas, como uma tentativa de aumentar o rendimento no futuro (Robb \& Robinson, 2014), mesmo controlando efeitos de variáveis perturbadoras (ano, estado e crescimento da população). Dessa maneira, corrobora-se também que o objetivo máximo da criação de empresa é o aumento da riqueza de quem empreende no nível regional ou nacional e não apenas no nível individual, proposta defendida por Porter and Kramer (2011). Esse efeito é visto na realidade brasileira tanto a curto, médio e longo prazo.

Nas situações em que a criação de empresa é causa do desenvolvimento, se incentivado, aumenta-se a quantidade de famílias de classe média e alta e diminui-se a quantidade de famílias de classe baixa. Assim, as novas empresas parecem ser também motoras da prosperidade social (Stinchcombe, 2000). Elas contratam trabalhadores, usam tecnologias, precisa de população previamente (ou concomitantemente) capacitada para exercerem atividades, e simultaneamente usam capital e movimentam o mercado financeiro (Coelho, 2014; Kirzner, 1978; Tigre, 2006). Apesar desse processo não ter sido testado nessa pesquisa, a curto e médio prazo é o que aparentemente ocorre de acordo com os resultados da presente pesquisa. Neste caso, suas consequências são o desenvolvimento da sociedade, pelo menos no exemplo brasileiro.

Evidencia-se também que a taxa de criação de empresas aumenta o rendimento da população economicamente ativa, em especial, as sociedades limitadas e as anônimas. Isso corrobora com proposta de Porter and Kramer (2011) sobre a criação de valor na sociedade, mas especifica como isso ocorre no Brasil. Assim, uma região ou uma nação que possui muitas novas empresas há chances de ter maiores rendimentos da população em relação aos tempos prévios. A apropriação do valor das empresas (R. P. de Brito \& L. A. L. Brito, 2012) geralmente é feita pelos acionistas, empresários ou investidores. Eles contratam pessoas, passando então a dotar de renda e assim aumenta-se o rendimento geral da nação. Portanto, o alcance da maximização da renda é um fim que parece aumentar o desenvolvimento da sociedade por mecanismos indiretos empresariais. No entanto, aumentos no rendimento estão associados 
subsequentemente à baixa taxa de criação de empresa. Assim, alto rendimento é sinal de prosperidade econômica, mas é um inibidor de novas empresas, sendo sua fonte de instabilidade, explicando porque países ou regiões com maior desenvolvimento possui menores taxas de empreendedorismo (Souza \& Lopez, 2011). Ao todo, evidencia-se um ciclo que, se devidamente incentivado, gera um desenvolvimento e se desestimulado gera um retrocesso (ou subdesenvolvimento) socioeconômico.

\section{CONCLUSÃO}

O objetivo deste artigo foi analisar os efeitos mútuos entre o crescimento de novas empresas (total e tipos jurídicos de empresas) e o desenvolvimento da sociedade brasileira (classe baixa, classe média \& alta e rendimento da PEA) em um estudo longitudinal com dados estaduais e anuais. Dentro do mesmo estado, os resultados demonstram que (1) a elasticidade do rendimento sobre a criação de novas empresas é um padrão incentivador do desenvolvimento socioeconômico, (2) o aumento da quantidade de família de classe média e alta e a redução da classe baixa, incentivam a criação de novas empresas, especialmente as cooperativas, (3) há efeitos de curto (T) e longo prazo (até T-4) das características socioeconômicas da população sobre a criação de novas empresas, (4) a criação de novas empresas, em especial LTDA e S.A., aumentam o rendimento da população e a quantidade de famílias de classe média e alta em cada estado ao longo do tempo, (5) a criação de novas empresas, especialmente a LTDA, diminui a quantidade de família de classe baixa, (6) há efeitos de curto (T) e longo prazo (até T-4) da criação de novas empresas sobre as características socioeconômicas da população e (7) a elasticidade do desenvolvimento socioeconômico geralmente é maior na criação de novas empresas do que destas no desenvolvimento socioeconômico. Assim, há um ciclo de desenvolvimento socioeconômico no contexto brasileiro: baixo volume da classe baixa ou baixo rendimento da população e alto volume das classe média \& alta gera $(\rightarrow)$ criação de novas empresas e esta gera $(\rightarrow)$ maior volume de classe média \& alta ou maior rendimento e menor volume de classe baixa.

Como limitações, esta pesquisa enfrentou a dificuldade em obter dados secundários públicos que não são amplamente divulgados, não tendo acesso em todos os períodos dos estados brasileiros. Ademais, a tipologia Eireli não foi averiguada devido ao seu pouco tempo de registro. Como os resultados dependem da confiabilidade dos dados secundários do IBGE e das Juntas Comerciais, a fidedignidade das relações aqui encontradas precisam de cautela para 
fins de tomada de decisão. Não foram analisadas todas as dimensões do desenvolvimento socioeconômico brasileiro, como por exemplo, indicadores de saneamento e domicílios e de saúde, além de políticas públicas de desenvolvimento e do índice de desenvolvimento humano (IDH), constituindo assim mais uma limitação.

Aconselha-se promover como agenda de pesquisa, futuras investigações sobre como as empresas são impactadas internamente pelas características da demanda e como suas estruturas e estratégias promovem modificações no ambiente com um fim macroeconômico (agregado no desenvolvimento da nação). Pesquisas brasileiras podem ser feitas com dados agregados das atividades empresariais ou construir um painel de empresas em cada região e averiguar possíveis efeitos mútuos que gerem o desenvolvimento ou o subdesenvolvimento de uma nação.

* Esta pesquisa teve o apoio do Conselho Nacional de Desenvolvimento Científico e Tecnológico do Brasil - CNPq.

\section{REFERÊNCIAS}

Almeida, J. G., Santos, E. J. R., Albuquerque, C. P., \& Ferreira, J. A. (2013). Desemprego e empreendedorismo: da ambiguidade conceitual à eficácia das práticas de intervenção social. PLURAL, 20(1), 31-56.

Ballinger, G. A. (2004). Using generalized estimating equations for longitudinal data analysis. Organizational Research Methods, 7(2), 127-150.

Bielschowsky, R. (2012, dezembro). Estratégia de desenvolvimento e as três frentes de expansão no Brasil: um desenho conceitual. Economia e Sociedade, 21(especial), 729-747.

Braga, T. V. M. (2012, agosto/dezembro) A nova classe média no Brasil: reflexões acerca de suas expectativas. Gestão \& Sociedade, 1(2), 1-11.

Campos, T. M., Martens, C. D. P., Resende, M. R. de, Carmona, V. C., \& Lima, E. (2013). Produção científica brasileira sobre empreendedorismo social entre 2000 e 2012. Revista de Empreendedorismo e Gestão de Pequenas Empresas, 1(2), 60-89.

Coelho, F. U. (2014). Manual de direito comercial: Direito de empresa. São Paulo: Saraiva.

Cucculelli, M. (2012). Family firms, entrepreneurship and economic development. Economia Marche Journal of Applied Economics, 31(2), 1-8.

Davidsson, P. (2006). Nascent entrepreneurship: empirical studies and developments. Foundations and Trends in Entrepreneurship, 2(1), 1-76. 
Brito, R. P. de, \& Brito, L. A. L. (2012). Vantagem Competitiva e sua relação com o desempenho: uma abordagem baseada em valor. Revista de Administração Contemporânea, $16(3), 360-380$.

Faria, M. D., \& Carvalho, J. L. F. S. (2011, setembro/dezembro). Planejamento de cenários e amplificação da variabilidade da demanda. Revista Eletrônica de Administração, 17(3), 823846.

Ferro, J. R., \& Torkomian, A. L. V. (1988). A criação de pequenas empresas de alta tecnologia. Revista de Administração de Empresas, 28(2), 43-50.

Fochezatto, A. (2011, janeiro/abril). Estrutura da demanda final e distribuição de renda no Brasil: uma abordagem multissetorial utilizando uma matriz de contabilidade social. Economia, 12(1), 111-130.

Garofoli, G. (1994). New firm formation and regional development: the Italian case. Regional Studies, 28(4), 381-393.

Gartner, W. B. (1985). A conceptual framework for describing the phenomenon of new venture creation. The Academy of Management Review, 10(4), 696-706.

Haug, M. R. (1977). Measurement in social stratification. Annual Review of Sociology, 3, $51-77$.

Instituto Brasileiro de Geografia e Estatística (IBGE). (2013). Séries estatísticas. Recuperado de http://seriesestatisticas.ibge.gov.br/lista_tema.aspx?op=0\&no=6\#

Kerlinger, F. N. (1973). Foundations of behavioral research. New York, NY: Holt, Rinehart and Winston.

Kirzner, I. M. (1978). Competition and entrepreneurship. Chicago: University of Chicago Press.

Kuznets, S. (1955). Economic growth and income inequality. The American Economic Review, 45(1), 1-28.

Leff, N. H. (1979). Entrepreneurship and economic development: the problem revisited. Journal of Economic Literature, 17(1), 46-64.

Liedholm, C. (2002). Small firm dynamics: evidence from Africa and Latin America. Small Business Economics, 18(1-3), 225-240.

Liedholm, C. E., \& Mead, D. C. (2013). Small enterprises and economic development: The dynamics of micro and small enterprises. New York: Routledge.

Lins, A. G., Domingues, I. C. C., Del Corso, J. M., \& Costa, J. A. S. (2012, julho/dezembro). Relacionamento entre performance, estratégia, ambiente empresarial e finanças corporativas: um estudo empírico no mercado brasileiro. Revista Capital Científico, 10(2), 49-62. 
Martins, R. C. A. (2009). Cooperativas sociais no Brasil: debates e práticas na tecitura de um campo em construção. Sociedade e Estado, 24(3), 900-901.

Metcalfe, J. S. (2004). The entrepreneur and the style of modern economics. Journal of Evolutionary Economics, 14(2), 157-175.

Neri, M. C. (2011). Desigualdade de renda na década. Rio de Janeiro: FGV CPS.

Piff, P. K., Kraus, M. W., Côté, S., Cheng, B. H., \& Keltner, D. (2010). Having less, giving more: the influence of social class on prosocial behavior. Journal of Personality and Social Psychology, 99(5), 771-784.

Pinheiro, F. G. (2011, agosto). Empresa individual de responsabilidade limitada. Conteúdo Jurídico. Brasília/DF. Recuperado em 20 março, 2013, de http://www.conteudojuridico.com.br/?artigos\&ver=2.32963\&seo=1

Porter, M. E., \& Kramer, M. R. (2011, January/February). Creating shared value. Harvard Business Review, 89(1/2), 62-77.

Reynolds, P., \& Miller, B. (1992). New firm gestation: conception, birth, and implications for research. Journal of Business Venturing, 7(5), 405-417.

Robb, A. M., \& Robinson, D. T. (2014). The capital structure decisions of new firms. The Review of Financial Studies, 27(1), 153-179.

Romero, I. (2011). Analysing the composition of the SME sector in high-and low-income regions: some research hypotheses. Entrepreneurship \& Regional Development, 23(7-8), 637660.

Scarpin, M. R. S., Roncon, A., Correia, R. B., \& Hoeltgebaum, M. (2012). Proposta de indicadores para um observatório de empreendedorismo no Brasil. Estratégia \& Negócios, 5(3), 90-121.

Sobottka, E. A., Santos, H. P., Filho, Timm, L. B., Andrade, F., Grijó, E., Carneiro, C., Freitas, F. L. C., Martins, E. D. G., Tavares, T., Boza, G., \& Brendler, G. (2010). A junta comercial e o seu papel no desenvolvimento da economia (Resumo do Projeto de Pesquisa apresentado ao Ministério da Justiça/PNUD, no Projeto Pensando o Direito PRODOC BRA/07/004). Série Pensando o Direito, (28), Porto Alegre/Brasília: PUC/RS/ Ministério da Justiça. Recuperado de http://pensando.mj.gov.br/wpcontent/uploads/2015/07/28Pensando_Direito3.pdf

Souza, E. C. L., \& Lopez, G. S., Jr. (2011). Empreendedorismo e desenvolvimento: uma relação em aberto. Revista de Administração e Inovação, 8(3), 120-140.

Stinchcombe, A. L. (2000). Social structure and organizations. Advances in Strategic Management, 17, 229-259.

Storey, D. J. (1991). The birth of new firms: does unemployment matter? A review of the evidence. Small Business Economics, 3(3), 167-178. 
Suzuki, L. A., \& Patricoski, M. P. (2013). Psychological assessment: social class, socioeconomic status counseling. In W. M. Liu (Ed.). The Oxford handbook of social class in counseling (Chap. 14). New York: Oxford University Press.

Tigre, P. (2006). Gestão da inovação: A economia da tecnologia no Brasil. Rio de Janeiro: Elsevier Brasil.

Tomazette, M. (2011). Curso de direito empresarial: Teoria geral e direito societário (3a ed.). São Paulo: Atlas.

Vicenzi, S. E., \& Bulgacov, S. (2013, abril). Fatores motivadores do empreendedorismo e as decisões estratégicas de pequenas empresas. Revista de Ciências da Administração, 15(35), 208-221.

Wiklund, J., Davidsson, P., Audretsch, D., \& Karlsson, C. (2011). The future of entrepreneurship research. Entrepreneurship Theory and Practice, 35(1), 1-9. 